\title{
Differential expression of heterogenous nuclear ribonucleoproteins in viral co-infections.
}

Shahan Mamoor, MS 1

1shahanmamoor@gmail.com

East Islip, NY 11730

Co-infection is a phenomena by which a host or cell is infected by at least two pathogens ${ }^{1,2}$. The severe acute respiratory syndrome coronavirus 2 , SARS-CoV-2, has resulted in the death of greater than 200,000 people in the United States in less than one year, with an impending influenza season, raising the prospect of co-infection-related complications $s^{3,4,5}$. We mined a published microarray dataset ${ }^{6}$ to discover genes associated with viral co-infection in the blood of human patients, including co-infections involving human rhinovirus, enterovirus, respiratory syncytial virus A, human coronavirus HKU1 and influenza B. We found significant differential expression of heterogenous nuclear ribonucleoproteins including hnRNP K in the blood of patients with viral co-infections. These data suggest hnRNPs may be of relevance to immunity against viral co-infections or utilized by viruses during completion of the viral life cycle in the context of co-infection.

Keywords: viral co-infection, coronavirus, HCoV HKU1, SARS-CoV-2, systems biology of viral infection. 
The most basic relationship between host and pathogen is a simple infection between one pathogen and one host or cell. Re-infection, or a second infection of a host with the same pathogen despite an initial immune response can occur ${ }^{7}$. Super-infection, a process by which a bacterial infection complicates an existing viral infection, such as those found in the respiratory tract, can occur 8 . . A third type of complex infection setting is known as co-infection, a process by which a cell or organism already infected with a virus is then infected with a second, different virus ${ }^{1,2}$. The novel coronavirus SARS-CoV-2 has resulted in the death of over 200,000 Americans in less than one year ${ }^{3,4}$. The upcoming influenza season raises the prospect of complications resulting from Influenza and SARS-CoV-2 co-infection. A recent study of patients in Northern California found that of 116 patients testing positive for SARS-CoV-2, 24 (20.7\%) possessed a co-infection ${ }^{5}$. Little is known about the transcriptional changes that accompany coronavirus co-infection. We mined published microarray data 6 to understand in an unbiased fashion and at the systems-level the most significant gene expression changes in the blood of patients with viral co-infections, including viral co-infections involving a human coronavirus. We observed significant transcriptional changes in expression of multiple heterogenous nuclear ribonucleoproteins (hnRNPs), including hnRNP K, representing some of the largest gene expression changes transcriptome-wide in the blood of patients with viral co-infection.

\section{Methods}

We used dataset GSE $68310^{6}$ for this differential gene expression analysis of the blood of patients with viral co-infections. GSE68310 was generated using Illumina HumanHT-12 V4.0 
expression beadchip technology; analysis was performed with platform GPL10558. In the study from which this microarray data was obtained, investigators collected peripheral blood from patients at baseline, day 0 of infection, correlating with a visit to the clinic upon infection with a respiratory illness; day 2, day 4, day 6, and day 21 of infection following the clinic visit, as well as in the season following infection ("Spring"). We compared the blood of $n=2$ patients with HCoV HKU1 and human rhinovirus co-infection (IDs: JR0207 and JR1702) at baseline, day 0, day 2 , day 4 , day 6 , and day 21 . We compared the blood of $n=1$ patient with respiratory syncytial virus A (RSVA) virus infection (ID: JR0547) to the blood of $n=1$ patient with RSVA and rhinovirus co-infection (ID: JR1145). We compared the blood of $n=1$ patient with enterovirus infection (ID: JR0471) to the blood of $n=1$ patient with enterovirus and rhinovirus infection (ID: JR0209). We compared the blood of $n=4$ patients with influenza A infection only (JR0021, JR0023, JR0025 and JR0053) to the blood of $n=4$ patients with co-infection of influenza A and human rhinovirus (IDs: JR0033, JR0147, JR0174, JR0199). We compared the blood of $n=3$ patients with influenza B infection only (IDs: JR1412, JR1491, and JR1939) to the blood of $n=3$ patients with co-infection of influenza B and human rhinovirus (IDs: JR1053, JR1120 and JR1275). We compared the blood of patients with $n=6$ patients with rhinovirus infection only (IDs: JR0148, JR0154, JR0223, JR0228, JR0276, JR0287) at baseline, day 0, day 2, day 4, day 6, day 21 and Spring. We compared the blood of $n=3$ patients with influenza B infection only (IDs: JR1412, JR1491, JR1939) at baseline, day 0, day 2, day 4, day 6, day 21 and Spring. 
The Benjamini and Hochberg method of $p$-value adjustment was used for ranking of differential expression but raw $p$-values were used for assessment of statistical significance of global differential expression. Log-transformation of data was auto-detected, and the NCBI generated category of platform annotation was used. A statistical test was used to evaluate the significance of difference in hnRNP gene expression between the blood of a patient with a single infection and the blood of a patient or patient(s) with co-infection, or between the blood of patients with co-infection over time, using either a two-tailed, unpaired t-test with Welch's correction, or a one-way ANOVA with Dunnett's multiple comparisons test. All statistical tests were performed with PRISM 8.4.0 (455).

\section{Results}

We mined published microarray data 6 to discover genes associated with viral co-infection in the blood of humans during the course of bona fide infections in the clinic.

Co-infection of human coronavirus HKU1 and human rhinovirus is associated with differential expression of hnRNP K, hnRNP A0, and hnRNP A3.

By comparing the blood of two patients with infection of both HCoV HKU1 and human rhinovirus at baseline, day 0 , day 2 , day 4 , day 6 , and day 21 , we identified significant differential expression of hnRNP K and hnRNP A0 over the course of co-infection in patient blood (Table 1). When sorting each transcript measured by microarray based on significance of change in expression across time, hnRNP A0 ranked 201 out of 47254 total transcripts $(99.6 \%$ differential expression) while hnRNP K ranked 47 out of 47254 total transcripts $(99.9 \%$ 
differential expression) (Table 1). Differential expression of hnRNP K and hnRNP A0 was statistically significant (Table $1 ; p=0.0016619$ and $p=0.0066584$, respectively).

We obtained exact mRNA expression levels for each hnRNP to directly compare hnRNP expression over the course of co-infection. At day 2 and day 4, expression of hnRNP K was significantly decreased as compared to baseline, with a mean fold change of $0.9707 \pm 0.0021$ and $0.9763 \pm 0.0054$, respectively (Figure $1 ; p=0.0041$ at day 2 and $p=0.0117$ at day 4 , respectively) At day 0 and day 4, expression of hnRNP A0 was also significantly decreased as compared to baseline, with a mean fold change of $0.9684 \pm 0.0002$ and $0.9692 \pm 0.0028$, respectively (Table $1 ; p=0.0091$ for at day 0 and $p=0.0104$ at day 4$)$.

To a lesser extent, hnRNP A3 was also significantly differentially expressed over time in the blood of two patients with co-infection of HCoV HKU1 and human rhinovirus. When sorting each transcript measured by microarray based on significance of change in expression across time, hnRNP A3 ranked 1321 out of 47254 total transcripts (97.2\% differential expression) (Table 1). Differential expression of hnRNP A3 was statistically significant (Table 1; $p=0.0424469)$.

Co-infection of respiratory syncytial virus A (RSVA) and human rhinovirus is associated with differential expression of hnRNP L and hnRNP A2B1.

Next, we compared total gene expression in the blood of a patient with infection of RSVA with that of a patient with co-infection of RSVA and human rhinovirus and found significant differential expression of hnRNP L and hnRNP A2B1 (Figure 2). When sorting each 
transcript measured by microarray based on significance of change in expression between single infection and co-infection, hnRNP L ranked 63 out of 47254 total transcripts (99.9\% differential expression) while hnRNP A2B1 ranked 4095 out of 47254 total transcripts (91.3\% differential expression) (Table 2). Differential expression of hnRNP L and hnRNP A2B1 was statistically significant (Table $2 ; p=6.52 \mathrm{E}-07$ and $p=1.98 \mathrm{E}-02$, respectively).

We obtained exact hnRNP L mRNA expression levels to directly compare hnRNP L expression in RSVA infection to that of RSVA and human rhinovirus co-infection. hnRNP L was expressed at significantly lower levels in the blood of a patient with co-infection of RSVA and human rhinovirus as compared to RSVA infection only; this difference was statistically significant (Figure 2; $p<0.0001$ ). We calculated a mean fold change of $0.9398 \pm 0.0149$ in hnRNP L expression in RSVA and human rhinovirus co-infected patient blood relative to that of patient with RSVA infection only (Table 2).

Co-infection of enterovirus and human rhinovirus is associated with differential expression of hnRNP K, hnRNP R, hnRNP L, hnRNP M, and hnRNP UL1.

Next, we compared total gene expression in the blood of a patient with infection of enterovirus only with that of a patient with co-infection of enterovirus and human rhinovirus and found significant differential expression of hnRNP K, hnRNP R, hnRNP L, hnRNP M, and hnRNP UL1 (Table 3). When sorting each transcript measured by microarray based on significance of change in expression between single infection and co-infection, hnRNP K ranked 99 out of 47254 total transcripts (99.8\% differential expression), hnRNP R ranked 1661 out of 
47254 total transcripts (96.5\% differential expression), hnRNP L ranked 1866 out of 47254 total transcripts (96.1\% differential expression), hnRNP M ranked 2412 out of 47254 total transcripts (94.9\% differential expression), and hnRNP UL1 ranked 4177 out of 47254 total transcripts (91.2\% differential expression) (Table 3). Differential expression of hnRNP K, hnRNP R, hnRNP L, hnRNP M, and hnRNP UL1 in enterovirus and human rhinovirus co-infection was statistically significant (Table 3; $p=1.47 \mathrm{E}-07$ for hnRNP K, $p=1.46 \mathrm{E}-03$ for hnRNP R, $p=$ 2.05E-03 for hnRNP L, $p=4.41 \mathrm{E}-03$ for hnRNP M, and $p=1.75 \mathrm{E}-02$ for hnRNP UL1 respectively).

We obtained exact hnRNP K mRNA expression levels to directly compare hnRNP K expression in the blood in enterovirus infection to that of a patient with co-infection of enterovirus and human rhinovirus. hnRNP K was expressed at significantly higher levels in the blood of a patient with co-infection of enterovirus and human rhinovirus as compared to enterovirus infection only; this difference was statistically significant (Figure $3 ; p<0.0001$ ). We calculated a mean fold change of $1.0814 \pm 0.0153$ in hnRNP K expression in enterovirus and human rhinovirus co-infected patient blood relative to enterovirus-only infected patient blood (Table 3).

Co-infection of influenza $A$ and human rhinovirus is associated with differential expression of hnRNP A1, hnRNP D, hnRNP M, hnRNP AB, hnRNP H1, hnRNP A0, and hnRNP R.

To determine if hnRNPs were differentially expressed during influenza co-infection, we compared total gene expression in the blood of four patients with influenza A infection with that of four patients with co-infection of influenza A and human rhinovirus. We identified significant 
differential expression of seven different hnRNPs in the blood of patients with influenza A and human rhinovirus co-infection (Table 4): this included hnRNP A1, hnRNP D, hnRNP M, hnRNP AB, hnRNP H1, hnRNP A0, and hnRNP R. When sorting all transcripts based on significance of difference in expression between the blood of patients with influenza A and human rhinovirus infection as compared to that of patients with influenza A infection only, hnRNP A1 ranked 404 out of 47254 total transcripts (99.1\% differential expression), hnRNP D ranked 1159 out of 47254 total transcripts (97.5\% differential expression), hnRNP M ranked 1248 out of 47254 total transcripts (97.4\% differential expression), hnRNP AB ranked 1923 out of 47254 total transcripts (95.9\% differential expression), hnRNP H1 ranked 1937 out of 47254 total transcripts (95.9\% differential expression), hnRNP A0 ranked 2059 out of 47254 total transcripts $(95.6 \%$ differential expression), and hnRNP R ranked 2569 out of 47254 total transcripts (94.6\% differential expression). Differential expression of hnRNP A1, hnRNP D and hnRNP M, hnRNP AB, hnRNP H1, hnRNP A0, and hnRNP R in influenza A and human rhinovirus co-infection was statistically significant ( $p=2.96 \mathrm{E}-07$ for hnRNP A1, $p=5.89 \mathrm{E}-05$ for hnRNP D, $p=8.42 \mathrm{E}-05$ for hnRNP M, $p=4.56 \mathrm{E}-04$ for hnRNP AB, $p=4.68 \mathrm{E}-04$ for hnRNP H1, $p=5.79 \mathrm{E}-04$ for hnRNP A0, and $p=1.26 \mathrm{E}-03$ for hnRNP R).

Co-infection of influenza B and human rhinovirus is associated with differential expression of hnRNP DL, hnRNP A0 and hnRNP K.

Finally, we also compared total gene expression in the blood of three patients with influenza B single infection with that of three patients with influenza B and human rhinovirus 
co-infection, finding that hnRNP DL, hnRNP A0 and hnRNP K were each among the genes most differentially expressing during influenza B and human rhinovirus co-infection (Table 5). When sorting each transcript measured by microarray based on significance of change in expression between single infection and co-infection, hnRNP DL ranked 472 out of 47254 total transcripts (99.0\% differential expression), hnRNP A0 ranked 1011 out of 47254 total transcripts (97.9\% differential expression), and hnRNP K ranked 1723 out of 47254 total transcripts $(96.4 \%$ differential expression). Differential expression of hnRNP DL, hnRNP A0 and hnRNP K in influenza B and human rhinovirus co-infection was statistically significant $(p=1.36 \mathrm{E}-05$ for hnRNP DL, $p=1.09 \mathrm{E}-04$ for hnRNP A0, and $p=4.45 \mathrm{E}-04$ for hnRNP K, respectively).

Infection with human rhinovirus infection only is associated with differential expression of hnRNP DL, hnRNP A0, hnRNP UL1, hnRNP M, hnRNP R and hnRNPA2B1 but not hnRNP K, and infection with influenza $\mathrm{B}$ only is associated with less significant differential expression of hnRNP K.

To determine whether differential expression of hnRNPs was specific to viral coinfection, we examined differential expression of hnRNPs in six patients with infection of human rhinovirus only. We observed significant differential expression of multiple hnRNPs, including hnRNP DL (Figure 4), as well as several other hnRNPs that were found to be differentially expressed in the context of viral co-infection, including hnRNP A0, hnRNP UL1, hnRNP M, hnRNP R, and hnRNP A2B1. However, hnRNP K, an hnRNP differentially expressed in the context of multiple viral co-infections involving human rhinovirus, was not differentially expressed in patients with infection of human rhinovirus only (Table 6). Moreover, while 
hnRNP K differential expression could be observed in the blood of four patients over the course of influenza B infection only, it was both less significant (Table 7; $p=3.47 \mathrm{E}-02$ ) and less differential (Table 7; rank 6830 out of 47254 transcripts, or $85.5 \%$ differential expression) than in co-infection of influenza B and human rhinovirus (Table 5; rank 1723 out of 47254 transcripts, or $96.4 \%$ differential expression; $p=4.45 \mathrm{E}-04)$. We conclude that differential expression of hnRNP K may be a specific feature of viral co-infections.

\section{Discussion}

We found significant differential expression of multiple heterogenous nuclear ribonucleoproteins (hnRNPs) in human blood, in several viral co-infections including in a patient with viral co-infection of human rhinovirus and human coronavirus HKU1. Of all differentially expressed hnRNPs identified, differential expression of hnRNP K appeared to be most specific to viral co-infection as opposed to being a feature of viral infection in general. hnRNP K is phosphorylated by ERK on serine residues 284 and 353, leading to its localization in the cytoplasm, and mutation of these residues results in inability of hnRNP K to accumulate in the cytoplasm and function in translational regulation, inhibiting translation of messenger RNAs containing a $3^{\prime}$ differentiation-control element (DICE) ${ }^{10}$. In the context of some viral infections, hnRNP K has been described as an interaction partner of viral proteins or important for the viral life cycle. hnRNP K can interact with the core protein of the dengue virus ${ }^{11}$, as well as with the HIV-1 Nef protein ${ }^{12}$. Interaction of hnRNP K with Nef is positively associated with transcription of HIV-1 viral genes through Tat ${ }^{12}$. 
Involvement of hnRNPs in the molecular biology of the coronavirus family has been described ${ }^{13}$ this includes interaction between the SARS-CoV-1 nucleocapsid protein and hnRNP A $1{ }^{14}$, as well as interaction of hnRNP Q with the $3^{\prime}$ end of the transmissible gastroenteritis coronavirus (TGEV) genome. Depletion of hnRNP Q resulted in decreased transcription of TGEV viral RNAs ${ }^{15}$.

By mining published microarray data ${ }^{6}$, we found specific differential expression of hnRNP K in human blood in multiple viral co-infections including human coronavirus HKU1 and human rhinovirus co-infection, human rhinovirus and influenza B co-infection, as well as in human rhinovirus and enterovirus co-infection. hnRNP K and other heterogenous ribonucleoproteins may be relevant to fundamental transcriptional processes underlying infection of a host or cell with more than one virus. 


\section{References}

1. Alter, M.J., 2006. Epidemiology of viral hepatitis and HIV co-infection. Journal of hepatology, 44, pp.S6-S9.

2. Heng, M.C., Heng, S.Y. and Allen, S.G., 1994. Co-infection and synergy of human immunodeficiency virus-1 and herpes simplex virus-1. The Lancet, 343(8892), pp.255-258.

3. Andersen, K.G., Rambaut, A., Lipkin, W.I., Holmes, E.C. and Garry, R.F., 2020. The proximal origin of SARS-CoV-2. Nature medicine, 26(4), pp.450-452.

4. World Health Organization. United States of America Situation. https://covid19.who.int/ region/amro/country/us.

5. Kim, D., Quinn, J., Pinsky, B., Shah, N.H. and Brown, I., 2020. Rates of co-infection between SARS-CoV-2 and other respiratory pathogens. Jama.

6. Zhai, Y., Franco, L.M., Atmar, R.L., Quarles, J.M., Arden, N., Bucasas, K.L., Wells, J.M., Nino, D., Wang, X., Zapata, G.E. and Shaw, C.A., 2015. Host transcriptional response to influenza and other acute respiratory viral infections-a prospective cohort study. PLoS Pathog, 11(6), p.e1004869.

7. Lambers, F.A., Prins, M., Thomas, X., Molenkamp, R., Kwa, D., Brinkman, K., van der Meer, J.T., Schinkel, J. and MOSAIC (MSM Observational Study of Acute Infection with Hepatitis C) Study Group, 2011. Alarming incidence of hepatitis $\mathrm{C}$ virus re-infection after treatment of sexually acquired acute hepatitis $\mathrm{C}$ virus infection in HIV-infected MSM. Aids, 25(17), pp.F21-F27.

8. Hament, J.M., Kimpen, J.L., Fleer, A. and Wolfs, T.F., 1999. Respiratory viral infection predisposing for bacterial disease: a concise review. FEMS Immunology \& Medical Microbiology, 26(3-4), pp.189-195.

9. Navarini, A.A., Recher, M., Lang, K.S., Georgiev, P., Meury, S., Bergthaler, A., Flatz, L., Bille J., Landmann, R., Odermatt, B. and Hengartner, H., 2006. Increased susceptibility to bacterial superinfection as a consequence of innate antiviral responses. Proceedings of the National Academy of Sciences, 103(42), pp.15535-15539.

10.Habelhah, H., Shah, K., Huang, L., Ostareck-Lederer, A., Burlingame, A.L., Shokat, K.M., Hentze, M.W. and Ronai, Z.E., 2001. ERK phosphorylation drives cytoplasmic accumulation of hnRNP-K and inhibition of mRNA translation. Nature cell biology, 3(3), pp.325-330. 
11.Chang, C.J., Luh, H.W., Wang, S.H., Lin, H.J., Lee, S.C. and Hu, S.T., 2001. The heterogeneous nuclear ribonucleoprotein $\mathrm{K}$ ( $\mathrm{hnRNP \textrm {K }}$ ) interacts with dengue virus core protein. DNA and cell biology, 20(9), pp.569-577.

12.Wolf, D., Witte, V., Clark, P., Blume, K., Lichtenheld, M.G. and Baur, A.S., 2008. HIV Nef enhances Tat-mediated viral transcription through a hnRNP-K-nucleated signaling complex. Cell host \& microbe, 4(4), pp.398-408.

13.de Wilde, A.H., Snijder, E.J., Kikkert, M. and van Hemert, M.J., 2017. Host factors in coronavirus replication. In Roles of Host Gene and Non-coding RNA Expression in Virus Infection (pp. 1-42). Springer, Cham.

14.Luo, H., Chen, Q., Chen, J., Chen, K., Shen, X. and Jiang, H., 2005. The nucleocapsid protein of SARS coronavirus has a high binding affinity to the human cellular heterogeneous nuclear ribonucleoprotein A1. FEBS letters, 579(12), pp.2623-2628.

15.Galán, C., Sola, I., Nogales, A., Thomas, B., Akoulitchev, A., Enjuanes, L. and Almazán, F., 2009. Host cell proteins interacting with the $3^{\prime}$ end of TGEV coronavirus genome influence virus replication. Virology, 391(2), pp.304-314. 


\begin{tabular}{|r|l|l|l|l|l|l|l|}
\hline \multicolumn{1}{|l}{ Rank } & ID & p-value & t & B & FC & Gene & Gene name \\
\hline $\mathbf{6 3}$ & ILMN_2389582 & $6.52 \mathrm{E}-07$ & -8.62 & 6.41553 & $\begin{array}{l}0.9398 \pm \\
0.0149\end{array}$ & HNRNPL & $\begin{array}{l}\text { heterogeneous } \\
\text { nuclear } \\
\text { ribonucleoprotein L }\end{array}$ \\
\hline $\mathbf{4 0 9 5}$ & ILMN_3178792 & $1.98 \mathrm{E}-02$ & -2.64 & -3.69833 & & HNRNPA2B1 & $\begin{array}{l}\text { heterogeneous } \\
\text { nuclear } \\
\text { ribonucleoprotein } \\
\text { A2/B1 }\end{array}$ \\
\hline
\end{tabular}

Table 2: hnRNP L and hnRNPA2B1 are differentially expressed in the blood during coinfection of respiratory syncytial virus $A$ (RSVA) and human rhinovirus.

The rank of differential expression, probe ID, $p$-value with respect to global differential expression, $t$, a moderated $t$-statistic, $B$, the log-odds of differential expression between the two groups compared, fold change of hnRNP gene expression when comparing RSVA and human rhinovirus co-infected patient blood to that of a patient with infection of RSVA only, gene and gene name are listed in this chart. GEO2R provides exact mRNA expression levels only for the 250 most differentially expressed genes. 
1

2

3

4

5

6

7

8

9

10

11

12

13

14

15

16

17

18

19

20

21

22

23

24

25

26

27

28

\begin{tabular}{|r|l|l|l|l|l|l|l|}
\hline \multicolumn{1}{|r|}{ Rank } & ID & p-value & t & B & FC & Gene & Gene name \\
\hline $\mathbf{9 9}$ & ILMN_3179371 & $1.47 \mathrm{E}-07$ & 9.7220474 & 7.843739 & $\begin{array}{l}1.0814 \pm \\
0.0153\end{array}$ & HNRNPK & $\begin{array}{l}\text { heterogeneous nuclear } \\
\text { ribonucleoprotein K }\end{array}$ \\
\hline $\mathbf{1 6 6 1}$ & ILMN_2175894 & $1.46 \mathrm{E}-03$ & 3.9582065 & -1.200071 & & HNRNPR & $\begin{array}{l}\text { heterogeneous nuclear } \\
\text { ribonucleoprotein R }\end{array}$ \\
\hline $\mathbf{1 8 6 6}$ & ILMN_2389582 & $2.05 \mathrm{E}-03$ & -3.7852547 & -1.533925 & & HNRNPL & $\begin{array}{l}\text { heterogeneous nuclear } \\
\text { ribonucleoprotein L }\end{array}$ \\
\hline $\mathbf{2 4 1 2}$ & ILMN_1745385 & $4.41 \mathrm{E}-03$ & 3.3968987 & -2.285096 & & HNRNPM & $\begin{array}{l}\text { heterogeneous nuclear } \\
\text { ribonucleoprotein M }\end{array}$ \\
\hline $\mathbf{4 1 7 7}$ & ILMN_1690268 & $1.75 \mathrm{E}-02$ & 2.6966665 & -3.616366 & & HNRNPUL1 & $\begin{array}{l}\text { heterogeneous nuclear } \\
\text { ribonucleoprotein U like } \\
1\end{array}$ \\
\hline
\end{tabular}

Table 3: hnRNP K, hnRNP R, hnRNP L, hnRNP M, and hnRNP UL1 are differentially expressed in the blood during co-infection of enterovirus and human rhinovirus.

The rank of differential expression, probe ID, $p$-value with respect to global differential expression, $\mathrm{t}$, a moderated $\mathrm{t}$-statistic, $\mathrm{B}$, the log-odds of differential expression between the two groups compared, fold change of hnRNP gene expression when comparing enterovirus and human rhinovirus co-infected patient blood to that of a patient with infection of enterovirus only, gene and gene name are listed in this chart. GEO2R provides exact mRNA expression levels only for the 250 most differentially expressed genes. 


\begin{tabular}{|r|l|l|l|l|l|}
\hline \multicolumn{1}{|l|}{ Rank } & ID & p-value & F & Gene & Gene name \\
\hline $\mathbf{4 0 4}$ & ILMN_1663447 & $2.96 E-07$ & 11.6192 & HNRNPA1 & $\begin{array}{l}\text { heterogeneous nuclear } \\
\text { ribonucleoprotein A1 }\end{array}$ \\
\hline $\mathbf{1 1 5 9}$ & ILMN_2321451 & $5.89 E-05$ & 6.8933 & HNRNPD & $\begin{array}{l}\text { heterogeneous nuclear } \\
\text { ribonucleoprotein D }\end{array}$ \\
\hline $\mathbf{1 9 2 3}$ & ILMN_1745385 & $8.42 E-05$ & 6.6178 & HNRNPM & $\begin{array}{l}\text { heterogeneous nuclear } \\
\text { ribonucleoprotein M }\end{array}$ \\
\hline $\mathbf{1 9 3 7}$ & ILMN_3246409 & $4.68 E-04$ & 5.3598 & HNRNPH1 & $\begin{array}{l}\text { heterogeneous nuclear } \\
\text { ribonucleoprotein H1 (H) }\end{array}$ \\
\hline $\mathbf{2 0 5 9}$ & ILMN_1753279 & $5.795-04$ & 5.2107 & HNRNPA0 & $\begin{array}{l}\text { heterogeneous nuclear } \\
\text { ribonucleoprotein A/B } \\
\text { ribonucleoprotein A0 }\end{array}$ \\
\hline $\mathbf{2 5 6 9}$ & ILMN_3177285 & $1.26 E-03$ & 4.6797 & HNRNPR & $\begin{array}{l}\text { heterogeneous nuclear } \\
\text { ribonucleoprotein R }\end{array}$ \\
\hline
\end{tabular}

Table 4: hnRNPA1, hnRNP D, hnRNP M, hnRNP AB, hnRNP H1, hnRNP A0, and hnRNP $\underline{R}$ are differentially expressed in co-infection of influenza $A$ and human rhinovirus.

The rank of differential expression, probe ID, $p$-value with respect to global differential expression, $\mathrm{t}$, a moderated $\mathrm{t}$-statistic, $\mathrm{B}$, the log-odds of differential expression between the two groups compared, gene and gene name are listed in this chart. GEO2R provides exact mRNA expression levels only for the 250 most differentially expressed genes. 

during co-infection of influenza $B$ and human rhinovirus.

The rank of differential expression, probe ID, $p$-value with respect to global differential expression, F statistic, gene and gene name are listed in this chart.

\begin{tabular}{|r|l|l|l|l|l|}
\hline \multicolumn{1}{|l}{ Rank } & ID & p-value & F & Gene & Gene name \\
\hline $\mathbf{4 7 2}$ & ILMN_1653432 & $1.36 E-05$ & 10.48201 & HNRNPDL & $\begin{array}{l}\text { heterogeneous nuclear } \\
\text { ribonucleoprotein D like }\end{array}$ \\
\hline $\mathbf{1 0 1 1}$ & ILMN_1753279 & $1.09 E-04$ & 7.94119 & HNRNPAO & $\begin{array}{l}\text { heterogeneous nuclear } \\
\text { ribonucleoprotein A0 }\end{array}$ \\
\hline $\mathbf{1 7 2 3}$ & ILMN_3260017 & $4.45 E-04$ & 6.46004 & HNRNPK & $\begin{array}{l}\text { heterogeneous nuclear } \\
\text { ribonucleoprotein K }\end{array}$ \\
\hline
\end{tabular}

Table 5: hnRNP DL, hnRNP A0 and hnRNP K are differentially expressed in the blood 

rhinovirus infection.

The rank of differential expression, probe ID, $p$-value with respect to global differential expression, F statistic, fold change in hnRNP DL gene expression at each timepoint over the course of Rhinovirus infection as compared to baseline, gene and gene name are listed in this chart.

Day 0: $0.9865 \pm 0.161$

Day 4: $0.9940 \pm 0.0132$

Day 6: $1.0049 \pm 0.0152$

Day 21: $1.0258 \pm 0.0138$

Spring: $1.0258 \pm 0.0209$

\section{Table 6: Differential expression of hnRNP DL in the blood of patients with human}

\begin{tabular}{|r|l|l|l|l|l|l|}
\hline \multicolumn{1}{|c|}{ Rank } & ID & p-value & F & FC & Gene & Gene name \\
\hline 121 & ILMN_1653432 & $7.6 E-05$ & 6.69436 & Day 0: $0.9865 \pm 0.161$ & HNRNPDL & heterogeneous \\
& & & & Day 2: $0.9947 \pm 0.0139$ & & nuclear \\
& & & & Day 4: $0.9940 \pm 0.0132$ & & ribonucleoprotein D \\
& & & & Day 6: $1.0049 \pm 0.0152$ & & like \\
& & & & Day 21: $1.0258 \pm 0.0138$ & & \\
& & & & Spring: $1.0258 \pm 0.0209$ & & \\
\hline
\end{tabular}

19 25 


\begin{tabular}{|c|c|c|c|c|c|}
\hline Rank & ID & p-value & $\mathbf{F}$ & Gene & Gene name \\
\hline 481 & ILMN_3177285 & $1.38 \mathrm{E}-03$ & 4.6146 & HNRNPR & $\begin{array}{l}\text { heterogeneous nuclear } \\
\text { ribonucleoprotein } \mathrm{R}\end{array}$ \\
\hline 484 & ILMN_1753279 & $1.39 \mathrm{E}-03$ & 4.61024 & HNRNPAO & $\begin{array}{l}\text { heterogeneous nuclear } \\
\text { ribonucleoprotein A0 }\end{array}$ \\
\hline 565 & ILMN_3180966 & $1.81 \mathrm{E}-03$ & 4.43725 & HNRNPUL1 & $\begin{array}{l}\text { heterogeneous nuclear } \\
\text { ribonucleoprotein U like } 1\end{array}$ \\
\hline 1243 & ILMN_2321451 & 6.81E-03 & 3.58028 & HNRNPD & $\begin{array}{l}\text { heterogeneous nuclear } \\
\text { ribonucleoprotein D }\end{array}$ \\
\hline 1319 & ILMN_1791575 & 7.56E-03 & 3.51471 & HNRNPLL & $\begin{array}{l}\text { heterogeneous nuclear } \\
\text { ribonucleoprotein L like }\end{array}$ \\
\hline 1573 & ILMN_2334587 & 1.03E-02 & 3.32327 & HNRNPC & $\begin{array}{l}\text { heterogeneous nuclear } \\
\text { ribonucleoprotein } C(\mathrm{C} 1 / \mathrm{C} 2)\end{array}$ \\
\hline 1775 & ILMN_1745385 & $1.25 \mathrm{E}-02$ & 3.20232 & HNRNPM & $\begin{array}{l}\text { heterogeneous nuclear } \\
\text { ribonucleoprotein M }\end{array}$ \\
\hline 1936 & ILMN_2175894 & 1.43E-02 & 3.11784 & HNRNPR & $\begin{array}{l}\text { heterogeneous nuclear } \\
\text { ribonucleoprotein } \mathrm{R}\end{array}$ \\
\hline 2074 & ILMN_1751368 & 1.59E-02 & 3.05498 & HNRNPD & $\begin{array}{l}\text { heterogeneous nuclear } \\
\text { ribonucleoprotein D }\end{array}$ \\
\hline 3061 & ILMN_3273854 & 2.91E-02 & 2.68501 & HNRNPA2B1 & $\begin{array}{l}\text { heterogeneous nuclear } \\
\text { ribonucleoprotein A2/B1 }\end{array}$ \\
\hline 3540 & ILMN_1781764 & 3.54E-02 & 2.56698 & HNRNPH2 & $\begin{array}{l}\text { heterogeneous nuclear } \\
\text { ribonucleoprotein } \mathrm{H} 2\left(\mathrm{H}^{\prime}\right)\end{array}$ \\
\hline 3743 & ILMN_3246409 & $3.8 \mathrm{E}-02$ & 2.52445 & HNRNPH1 & $\begin{array}{l}\text { heterogeneous nuclear } \\
\text { ribonucleoprotein } \mathrm{H} 1(\mathrm{H})\end{array}$ \\
\hline 3798 & ILMN_3244579 & 3.89E-02 & 2.5101 & HNRNPCL3 & $\begin{array}{l}\text { heterogeneous nuclear } \\
\text { ribonucleoprotein C-like } 3\end{array}$ \\
\hline 10340 & ILMN_3179371 & $1.51 \mathrm{E}-01$ & 1.6914 & HNRNPK & $\begin{array}{l}\text { heterogeneous nuclear } \\
\text { ribonucleoprotein K }\end{array}$ \\
\hline
\end{tabular}

Table 7: Differential expression of hnRNPs, including hnRNP A0 and hnRNP M, but not hnRNP K over the course of infection with human rhinovirus.

The rank of differential expression, probe ID, $p$-value with respect to global differential expression, $\mathrm{F}$ statistic, gene and gene name are listed in this chart. 
Table 8: Differential expression of hnRNP K in patient blood over the course of influenza B infection.

The rank of differential expression, probe ID, $p$-value with respect to global differential expression, F statistic, gene and gene name are listed in this chart.

(11 25 
A
hnRNP K HCoV HKU1 and Rhinovirus

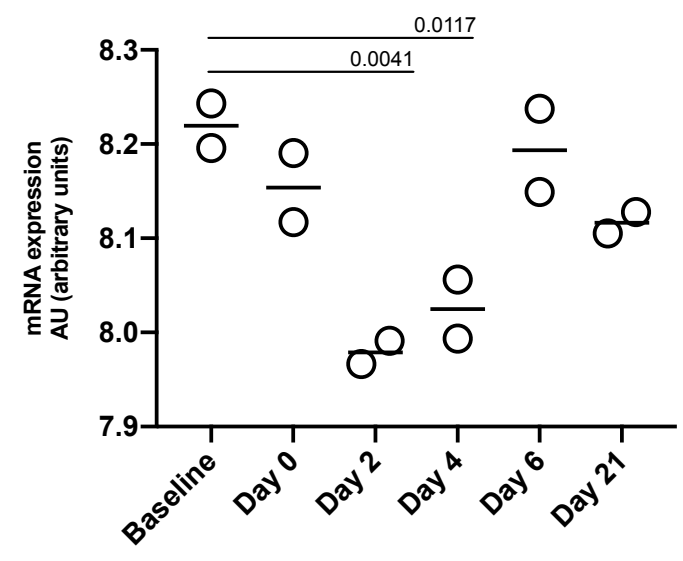

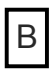

hnRNP A0

HCoV HKU1 and Rhinovirus

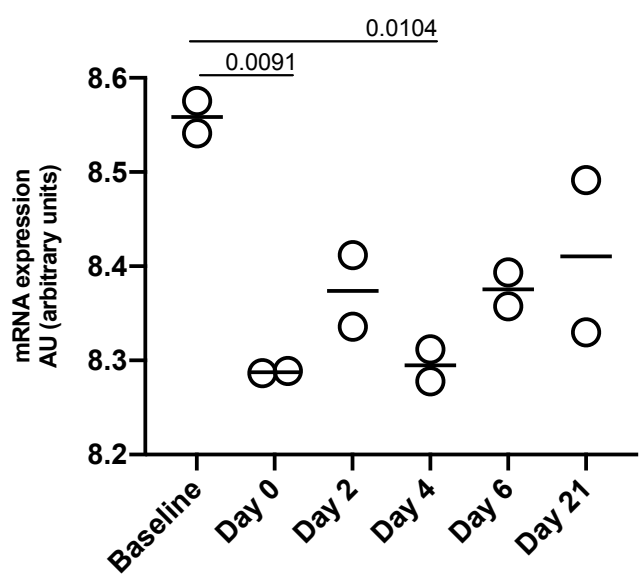

Figure 1: hnRNP K and hnRNP A0 are differentially expressed in the blood during human coronavirus HKU1 and human rhinovirus co-infection, and their expression significantly decreases during over the course of viral co-infection.

hnRNP K (A) and hnRNP A0 (B) gene expression levels are graphically represented in the blood of two patients with co-infection of HCoV HKU1 and human rhinovirus, with mean mRNA expression levels of hnRNP K and hnRNP A0 marked at each time point, and the result of a statistical test evaluating significance of difference in hnRNP gene expression as compared to baseline for each statistically significant time point. 
hnRNP L

Respiratory Syncytial Virus A (RSVA) vs. RSVA and Rhinovirus

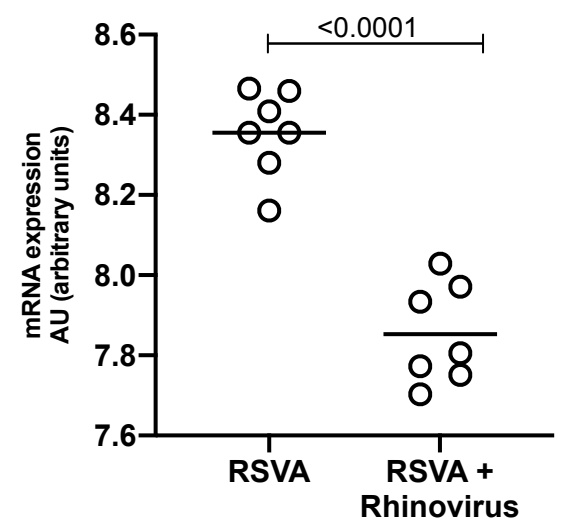

Figure 2: hnRNP L is differentially expressed in the blood during RSVA and human rhinovirus co-infection, at significantly lower levels as compared to RSVA infection only.

hnRNP L mRNA expression levels are graphically represented in the blood of a patient with RSVA infection only (left) and in the blood of a patient with co-infection of RSVA and rhinovirus (right), with mean mRNA expression level of hnRNP L marked, and the result of a statistical test evaluating significance of difference in hnRNP gene expression between coinfection and single infection listed above. 
Figure 3: hnRNP K is differentially expressed in the blood during enterovirus and human rhinovirus co-infection, at significantly higher levels as compared to enterovirus infection only.

hnRNP K mRNA expression levels are graphically represented in the blood of a patient with enterovirus infection only (left) and in the blood of a patient with co-infection of enterovirus and rhinovirus (right), with mean mRNA expression level of hnRNP K marked, and the result of a statistical test evaluating significance of difference in hnRNP gene expression between coinfection and single infection listed above. 


\section{hnRNP DL Rhinovirus infection}

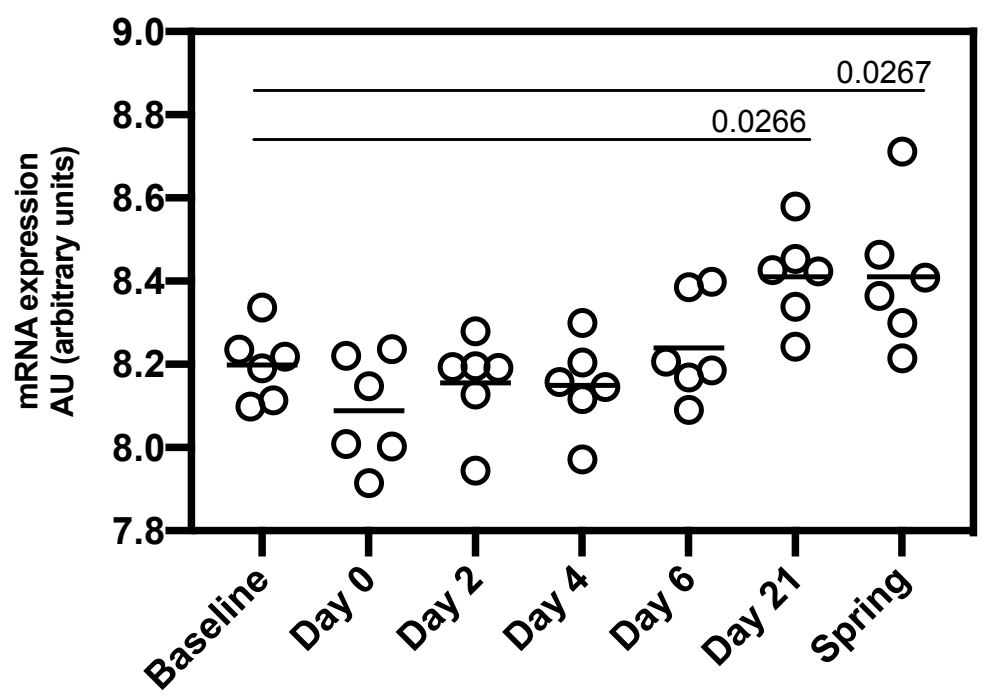

Figure 4: hnRNP DL is among the genes most differentially expressed in patient blood over the course of rhinovirus infection.

hnRNP DL mRNA expression levels are graphically represented in $n=6$ patients with infection of rhinovirus only, with mean hnRNP DL mRNA levels marked and with statistically significant changes in hnRNP DL gene expression relative to baseline listed above each time point as an exact $p$-value. 